City University of New York (CUNY)

CUNY Academic Works

2017

\title{
Economic Burden, Mortality, and Institutionalization in Patients Newly Diagnosed with Alzheimer's Disease
}

Christopher M. Black

Merck \& Co., Inc.

Howard Fillit

The Icahn School of Medicine at Mount Sinai

Lin Xie

STATinMED Research

Xiaohan $\mathrm{Hu}$

University of Southern California

M. Furaha Kariburyo

STATinMED Research

See next page for additional authors

\section{How does access to this work benefit you? Let us know!}

More information about this work at: https://academicworks.cuny.edu/ny_pubs/451

Discover additional works at: https://academicworks.cuny.edu

This work is made publicly available by the City University of New York (CUNY).

Contact: AcademicWorks@cuny.edu 


\section{Authors}

Christopher M. Black, Howard Fillit, Lin Xie, Xiaohan Hu, M. Furaha Kariburyo, Baishali M. Ambegaonkar, Onur Baser, Huseyin Yuce, and Rezaul K. Khandker 


\title{
Economic Burden, Mortality, and Institutionalization in Patients Newly Diagnosed with Alzheimer's Disease
}

\author{
Christopher M. Black ${ }^{\mathrm{a}, *}$, Howard Fillit ${ }^{\mathrm{b}}$, Lin Xie ${ }^{\mathrm{c}}, \mathrm{Xiaohan}_{\mathrm{Hu}}^{\mathrm{d}}$, M. Furaha Kariburyo ${ }^{\mathrm{c}}$, \\ Baishali M. Ambegaonkar ${ }^{\mathrm{a}}$, Onur Baser ${ }^{\mathrm{e}, \mathrm{f}}$, Huseyin Yuce ${ }^{\mathrm{g}}$ and Rezaul K. Khandker ${ }^{\mathrm{a}}$ \\ ${ }^{\mathrm{a}}$ Merck \& Co., Inc., Kenilworth, NJ, USA \\ ${ }^{\mathrm{b}}$ The Icahn School of Medicine at Mount Sinai, and the Alzheimer's Drug Discovery Foundation, \\ New York, NY, USA \\ ${ }^{\mathrm{c}}$ STATinMED Research, Ann Arbor, MI, USA \\ ${ }^{\mathrm{d}}$ University of Southern California, Los Angeles, CA, USA \\ ${ }^{\mathrm{e}}$ Department of Surgery, Center for Innovation and Outcomes Research, Columbia University, \\ New York, NY, USA \\ ${ }^{\mathrm{f}}$ STATinMED Research, New York, NY, USA

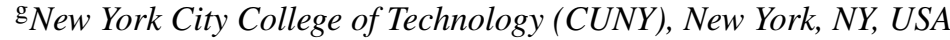

Handling Associate Editor: Helen Wu

Accepted 31 August 2017

\begin{abstract}
.
Background: Current information is scarce regarding comorbid conditions, treatment, survival, institutionalization, and health care utilization for Alzheimer's disease (AD) patients.

Objectives: Compare all-cause mortality, rate of institutionalization, and economic burden between treated and untreated newly-diagnosed AD patients.

Methods: Patients aged 65-100 years with $\geq 1$ primary or $\geq 2$ secondary AD diagnoses (ICD-9-CM:331.0] with continuous medical and pharmacy benefits for $\geq 12$ months pre-index and $\geq 6$ months post-index date (first AD diagnosis date) were identified from Medicare fee-for-service claims 01JAN2011-30JUN2014. Patients with AD treatment claims or AD/ADrelated dementia diagnosis during the pre-index period were excluded. Patients were assigned to treated and untreated cohorts based on AD treatment received post-index date. Total 8,995 newly-diagnosed AD patients were identified; 4,037 (44.8\%) were assigned to the treated cohort. Time-to-death and institutionalization were assessed using Cox regression. To compare health care costs and utilizations, $1: 1$ propensity score matching (PSM) was used.

Results: Untreated patients were older ( 83.85 versus 81.44 years; $p<0.0001)$, with more severe comorbidities (mean Charlson comorbidity index: 3.54 versus $3.22 ; p<0.0001$ ). After covariate adjustment, treated patients were less likely to die (hazard ratio $[\mathrm{HR}]=0.69 ; p<0.0001)$ and were associated with $20 \%$ lower risk of institutionalization $(\mathrm{HR}=0.801 ; p=0.0003)$. After PSM, treated AD patients were less likely to have hospice visits $(3.25 \%$ versus $9.45 \% ; p<0.0001)$, and incurred lower annual all-cause costs $(\$ 25,828$ versus $\$ 30,110 ; p=0.0162)$.

Conclusion: After controlling for comorbidities, treated AD patients have better survival, lower institutionalization, and sometimes fewer resource utilizations, suggesting that treatment and improved care management could be beneficial for newly-diagnosed AD patients from economic and clinical perspectives.
\end{abstract}

Keywords: Alzheimer's disease, institutionalization, Medicare, mortality

\footnotetext{
${ }^{*}$ Correspondence to: Christopher M. Black, MPH, Associate Director, Immunology \& Neuroscience, Center for Observational and Real-World Evidence (CORE), 126 E. Lincoln Ave., Mailstop:
}

RY32-211, Rahway, NJ 07065, USA. Tel.: +1 732594 3037; E-mail: Christopher.Black2@Merck.com. 


\section{INTRODUCTION}

Alzheimer's disease (AD) is characterized by a gradual decline in memory and cognitive skills that limit a patient's ability to perform daily activities $[1,2]$. An estimated 5.4 million US patients are currently diagnosed with $\mathrm{AD}$, with about 5 million patients over age 65 [2, 3]. Age, genetics, physical inactivity, unhealthy diet, and some cardiovascular diseases have been reported as significant risk factors of $\mathrm{AD}$ [1]. With the aging US population, $\mathrm{AD}$ prevalence is expected to increase to approximately 13 million by 2050 [2]. Currently, no cure for $\mathrm{AD}$ exists. Existing treatments focus on relieving symptoms and slowing symptom progression, maintaining cognitive and daily function, and managing behavioral symptoms [4]. So far, four medications (donepezil, rivastigmine, galantamine, and memantine) have been approved for $\mathrm{AD}$ management [2].

$\mathrm{AD}$ is also known to be one of the main causes of disability and institutionalization among the elderly population leading to significant economic burden $[5,6]$. The 2016 direct costs for all individuals diagnosed with $\mathrm{AD}$ were estimated at $\$ 221$ billion, including $\$ 150$ billion to Medicare and Medicaid [1]. Other studies examining the economic burden of AD have only assessed prevalent cases and have not compared the current economic burden among treated and untreated $\mathrm{AD}$ patients $[7,8]$. Due to a scarcity of information on specific AD-related outcomes, this study evaluated all-cause mortality, rate of institutionalization, and economic burden of treated versus untreated patients newly diagnosed with $\mathrm{AD}$ in the United States.

\section{METHODS}

\section{Data source}

A retrospective analysis was performed using a $5 \%$ random national sample of the Medicare administrative database and Minimum Data Set (MDS) from January 2010 through December 2014. The MDS data was linked to Medicare data to assess the overall health care utilization of $\mathrm{AD}$ patients. MDS data includes severity proxy measurements, such as the Cognitive Performance Scale (CPS), and the Activities of Daily Living (ADL) Dependency Scale, captured for patients with available MDS data.

\section{Patient identification}

\section{Primary analysis population}

Patients aged 65-100 years and with at least 1 primary or at least 2 secondary diagnoses for $\mathrm{AD}$ (International Classification of Disease 9th Edition Clinical Modification [ICD-9-CM] code: 331.0) during the identification period from January 2011 to June 2014 were included in the primary analysis cohort (Fig. 1). The first AD diagnosis date observed during the identification period was designated as the index date. Patients were also required to have continuous health plan enrollment with medical and pharmacy benefits for at least 12 months pre-index date (baseline period) and at least 6 months postindex date (follow-up period). Patient data were assessed until the earliest of death, health plan disenrollment, or the end of the study period.

Patients with diagnosis claims for AD, AD-related dementia (ICD-9-CM: 290.xx, 294.xx, 331.1, 331.2, $331.7,331.82,331.89,331.9,797)$, or pharmacy claims for an $\mathrm{AD}$ treatment (donepezil, rivastigmine, galantamine, and memantine) during the baseline period were excluded.

Patients were assigned to treated and untreated cohorts based on AD treatment prescriptions within 6 months of index AD diagnosis during the follow-up period.

\section{Economic analysis population}

Time-to-treatment analysis indicated that $10 \%$ of the primary analysis population did not receive their first AD treatment until 1 year or longer after their diagnosis. Therefore, a subset of patients from the primary analysis population was selected to analyze economic outcomes, excluding the $10 \%$ who received their first $A D$ treatment 1 year or more after $A D$ diagnosis. This subset of patients was required to 1) have continuous health plan enrollment with medical and pharmacy benefits for at least 12 months preindex date and at least 12 months post-index date; and 2) have received their first AD treatment within 6 months of their index AD diagnosis.

\section{Study variables}

\section{Outcomes variables for primary analysis population}

Overall survival and rate of institutionalization were the main end points of the primary analysis. Overall survival was defined as patients who did not die during the study period. Using the MDS data, 
patients who resided in a post-acute skilled nursing home (SNF) or nursing home for at least 120 consecutive days were considered institutionalized [9]. Mortality and institutionalization rates during the overall follow-up period were captured and estimated in 100 person-years.

\section{Outcomes variables for economic analysis}

All-cause and AD-related dementia health care resource use and costs were the main outcomes for economic analysis. Claims were attributed to AD-related dementia if they were associated with a primary or non-primary diagnosis of $\mathrm{AD}$ or AD-related dementia.

\section{Baseline variables}

Baseline socio-demographic characteristics included age, race, sex, and US geographical region. Clinical characteristics included Charlson Comorbidity Index (CCI) score [10] and individual comorbid conditions during the 12 months prior to the index date.

All-cause health care utilization and costs as well as a number of diagnostic tests (magnetic resonance imaging [MRI], positron emission tomography $[\mathrm{PET}]$, and computed tomography $[\mathrm{CT}]$ scans) were evaluated for the 12-month baseline period.

The CPS and ADL Dependency Scale were available for patients with linked MDS data. The CPS has five components that address cognitive and communication domains (memory, decision making, and making self-understood), presence of coma, and eating dependency [11]. CPS scores range from 0 (intact) to 6 (very severe impairment). The ADL-Short Form (SF) includes four items (eating, personal hygiene, toileting, and locomotion) [12]. The ADL-SF sums the individual ADL items (rating of 0 to 4 ) into a scale running from 0 to 16 .

\section{Statistical analysis}

All study variables, including baseline and outcome measures, were examined descriptively. Numbers and percentages were provided for dichotomous and polychotomous variables. Means and standard deviations were provided for continuous variables. For dichotomous and polychotomous variables, $p$-values were calculated according to the chi-square test, and for continuous variables, $t$-tests were used to calculate $p$-values.

In the primary analysis, a multivariable Cox proportional hazard model with time dependent and independent covariates was applied to estimate hazard ratio (HR) and 95\% confidence interval (CI) of overall survival and rate of institutionalization between treated and untreated $\mathrm{AD}$ patients. Time independent covariates included patients' socio-demographic and clinical characteristics. AD treatment was included in the Cox model as a timedependent covariate.

In the economic analysis, possible confounders of health care utilization and costs were controlled using $1: 1$ propensity score matching (PSM) between patients who received treatment within 6 months of $\mathrm{AD}$ diagnosis and untreated $\mathrm{AD}$ patients.

\section{RESULTS}

\section{Primary analysis}

\section{Patient characteristics}

A total of 59,921 Medicare beneficiaries aged between 65-100 years had an index diagnosis of AD between January 2011 and June 2014. From this sample, 50,926 patients were excluded because they had an $\mathrm{AD}$ or AD-related dementia diagnosis or were prescribed AD treatment during the baseline period. A total of 8,995 treatment naive and newly-diagnosed $\mathrm{AD}$ patients were selected for the primary analysis of mortality and institutionalization (treated: $n=4,037$; untreated: $n=4,958$; Fig. 1).

Approximately $44.8 \%$ of patients initiated treatment (average 122 days post-AD diagnosis); mean follow-up days for treated and untreated patients was 681 versus 794 days, $p<0.0001$. Treated patients were prescribed donepezil $(68.8 \%)$, memantine $(18.0 \%)$, rivastigmine $(12.1 \%)$, and galantamine $(1.2 \%)$ as their first treatment. Of newly-diagnosed AD patients, $74 \%$ were diagnosed in a physician's office or outpatient hospital, and $18.2 \%$ were diagnosed in an inpatient setting.

The untreated cohort included older patients (84.1 versus 81.6 years, $p<0.0001)$, with a greater proportion of Caucasian $(81.4 \%$ versus $80.4 \%, p=0.2384)$ and female patients $(75.7 \%$ versus $72.5 \%, p=0.0007)$ compared to the treated cohort (Supplementary Table 1). Supplementary Table 2 shows the baseline clinical characteristics of newly-diagnosed AD patients. At baseline, comorbid conditions such as congestive heart failure $(23.6 \%$ versus $14.6 \%$, $p<0.0001)$, peripheral vascular disease $(16.9 \%$ versus $13.1 \%, p<0.0001)$ and pneumonia $(9.9 \%$ versus $6.0 \%, p<0.0001)$ were generally more prevalent 


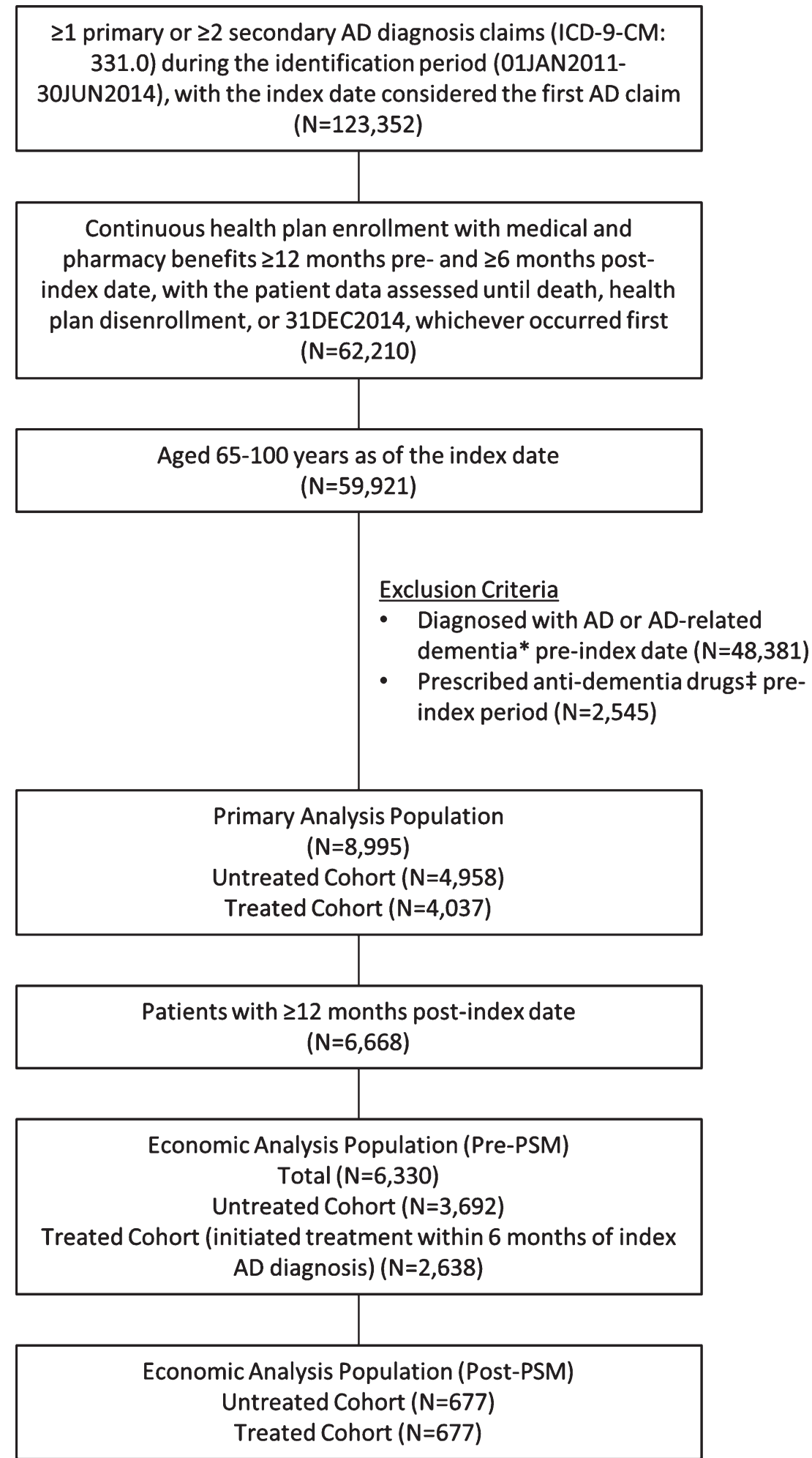

Fig. 1. Patient selection flow chart. AD, Alzheimer's disease; PSM, propensity score matching. *ICD-9-CM diagnosis claim for AD: 331.0; ICD-9-CM diagnosis claim for AD-related dementia: 290.xx, 294.xx, 331.1, 331.2, 331.7, 331.82, 331.89, 331.9, 797. ${ }^{\ddagger}$ donepezil, rivastigmine, galantamine, or memantine. 
Table 1

Cox model for time-to-death and time-to-institutionalization among newly-diagnosed AD patients (primary analysis population)

\begin{tabular}{|c|c|c|c|c|c|c|}
\hline \multirow[b]{2}{*}{ Covariates } & \multicolumn{3}{|c|}{ Time-to-Death } & \multicolumn{3}{|c|}{ Time-to-Institutionalization } \\
\hline & $\overline{\mathrm{HR}}$ & $(95 \% \mathrm{CI})$ & $p$-value & HR & $(95 \% \mathrm{CI})$ & $p$-value \\
\hline \multicolumn{7}{|c|}{ Index Treatment (Time-Dependent covariate) } \\
\hline \multicolumn{7}{|c|}{ Untreated (Reference) } \\
\hline Treated & 0.69 & $(0.63,0.75)$ & $<0.0001$ & 0.801 & $(0.71,0.903)$ & 0.0003 \\
\hline \multicolumn{7}{|l|}{ Time invariant covariate } \\
\hline \multicolumn{7}{|l|}{ Age Group } \\
\hline \multicolumn{7}{|l|}{ 65-74 (Reference) } \\
\hline $75-84$ & 1.76 & $(1.51,2.06)$ & $<0.0001$ & 1.154 & $(0.972,1.37)$ & 0.102 \\
\hline $85-94$ & 3.14 & $(2.71,3.65)$ & $<0.0001$ & 1.829 & $(1.549,2.159)$ & $<0.0001$ \\
\hline $95-100$ & 5.04 & $(4.206 .06)$ & $<0.0001$ & 1.983 & $(1.558,2.525)$ & $<0.0001$ \\
\hline \multicolumn{7}{|l|}{ Race } \\
\hline \multicolumn{7}{|l|}{ White (Reference) } \\
\hline Black & 0.78 & $(0.68,0.90)$ & 0.0005 & 1.021 & $(0.861,1.21)$ & 0.815 \\
\hline Hispanic & 0.69 & $(0.56,0.85)$ & 0.0004 & 0.571 & $(0.417,0.781)$ & 0.0005 \\
\hline Other (Asian/Native American) & 0.66 & $(0.53,0.83)$ & 0.0003 & 0.999 & $(0.767,1.302)$ & 0.9961 \\
\hline \multicolumn{7}{|l|}{ Gender } \\
\hline \multicolumn{7}{|l|}{ Male (Reference) } \\
\hline Female & 0.85 & $(0.77,0.93)$ & 0.0003 & 1.122 & $(0.989,1.272)$ & 0.0748 \\
\hline \multicolumn{7}{|l|}{ US Geographic Region } \\
\hline \multicolumn{7}{|l|}{ Northeast (Reference) } \\
\hline North Central & 1.15 & $(1.02,1.30)$ & 0.0257 & 1.031 & $(0.886,1.201)$ & 0.692 \\
\hline South & 1.15 & $(1.03,1.28)$ & 0.0134 & 0.902 & $(0.786,1.034)$ & 0.1392 \\
\hline West or Other & 1.14 & $(1.00,1.30)$ & 0.0431 & 0.595 & $(0.495,0.715)$ & $<0.0001$ \\
\hline \multicolumn{7}{|l|}{ Baseline Comorbid Conditions } \\
\hline CCI Score & 1.05 & $(1.03,1.06)$ & $<0.0001$ & 1.018 & $(0.999,1.038)$ & 0.0701 \\
\hline \multicolumn{7}{|l|}{ Individual Comorbidities } \\
\hline Parkinson's Disease & 1.01 & $(0.84,1.22)$ & 0.8964 & 0.956 & $(0.743,1.229)$ & 0.7238 \\
\hline Epilepsy & 1.08 & $(0.89,1.30)$ & 0.4323 & 1.097 & $(0.852,1.413)$ & 0.4732 \\
\hline Mood Disorder & 0.84 & $(0.76,0.93)$ & 0.0006 & 1.264 & $(1.115,1.434)$ & 0.0003 \\
\hline Pneumonia & 1.32 & $(1.16,1.50)$ & $<0.0001$ & 1.1 & $(0.908,1.334)$ & 0.3305 \\
\hline Mild Cognitive Impairment & 0.64 & $(0.48,0.86)$ & 0.0029 & 0.581 & $(0.404,0.834)$ & 0.0033 \\
\hline
\end{tabular}

Cox model with time-dependent covariates was performed among Untreated $n=4,958$ and Treated $n=4,037$ cohorts. HR, hazard ratio; CI, confidence interval; CCI, Charlson Comorbidity Index.

in the untreated cohort. In addition, the untreated cohort had higher mean CCI scores (3.54 versus $3.22, p<0.0001)$ and lower utilization of baseline tests including computed tomography (CT) scan (19.1\% versus $22.6 \%, p<0.0001)$ and magnetic resonance imaging (MRI) $(6.0 \%$ versus $12 \%, p<0.0001)$ compared to the treated cohort.

Approximately 1,915 AD patients resided in a SNF facility or nursing home during the baseline period. Untreated patients had higher baseline ADL scores of 8.8 versus $7.4(p<0.0001)$ and CPS scores of 3.2 versus $2.0(p<0.0001)$ indicating more significant impairment of daily function and cognition in comparison to treated patients. A lower proportion of patients in the untreated cohort had a diagnostic test such as a CT scan or MRI during the baseline period.

\section{Outcome: Mortality}

Untreated patients had a higher unadjusted incidence rate of death (18.9 versus 9.9 per 100 person-years, $p<0.0001)$. After covariate adjustment using Cox regression, patients were less likely to die if they had ever received an $\mathrm{AD}$ treatment (adjusted $\mathrm{HR}=0.69, p<0.0001$, Table 1 ).

\section{Outcome: Institutionalization}

A larger proportion of untreated patients were institutionalized at the time of diagnosis $(10.5 \%$ versus $4.3 \%, p<0.0001)$. After their index $\mathrm{AD}$ diagnosis, the untreated cohort had, on average, a shorter time-to-institutionalization period (188.5 versus 283.1 days, $p<0.0001$ ), and a higher rate of institutionalization compared to the treated cohort (11.0 versus 9.5 per 100 person-years, $p=0.0044$ ). After adjusting for baseline differences using Cox regression, patients who received $\mathrm{AD}$ treatment at any point were associated with a $20 \%$ lower risk of being institutionalized (adjusted HR $=0.80$, $p=0.0003$, Table 1). Older age and prior mood disorder diagnosis were risk factors associated with institutionalization. 


\section{Economic analysis}

\section{Patient characteristics}

A total of 6,668 patients were further selected from the primary analysis population if they had at least 12 months of follow-up. For the treatment cohort, the first $\mathrm{AD}$ treatment claim was required to have occurred within 6 months of the index AD diagnosis. A total of 6,330 patients were included in the final economic analysis population (treated cohort: $n=2,638$; untreated cohort: $n=3,692$; Fig. 1).

For patients in the treated cohort $(n=2,638)$, the average number of days to initiate the first $A D$ treatment was 33 days (median: 10 days). Treated patients were prescribed donepezil $(70.9 \%)$, memantine $(15.9 \%)$, rivastigmine $(11.9 \%)$, and galantamine $(1.3 \%)$ as their first treatment.

Untreated patients were older (83.6 versus 81.1 years, $p<0.0001)$ and had a higher proportion of women $(75.8 \%$ versus $72.6 \%, p=0.0049)$ than treated patients. Patients in the untreated cohort also had a higher prevalence of key individual comorbidities as well as higher CCI scores (3.5 versus $3.1, p<0.0001)$ compared to the treated cohort. The results were similar to those observed within the primary analysis population (Supplementary Tables 3 and 4).

During the 12-month baseline period, the untreated cohort had higher utilization for inpatient $(25.0 \%$ versus $18.4 \%, p<0.0001)$, post-acute SNF $(14.1 \%$ versus $6.8 \%, p<0.0001)$, home health agency ([HHA]; $19.3 \%$ versus $16.3 \%, p=0.0022$ ), and hospice visits $(3.1 \%$ versus $0.5 \%, p<0.0001)$, but lower utilization for outpatient office ( $86.1 \%$ versus $92.7 \%$, $p<0.0001)$ and outpatient hospital visits $(66.6 \%$ versus $73.3 \%, p<0.0001)$, compared to the treated cohort. The same trend was observed for health care costs.

\section{Economic outcomes}

A 1:1 PSM was used to adjust for baseline demographic and clinical characteristics between the treated and the untreated patients. A total of 677 patients were matched from each cohort with wellbalanced baseline characteristics.

Monthly trends in total Medicare expenditures spiked at the time of AD diagnosis (Fig. 2). The cost differences between treated and untreated patients were determined for the months leading up to the diagnosis and continued to increase throughout the span of the disease, especially in the first few months immediately after the diagnosis. In fact, during the month of diagnosis, untreated and treated $\mathrm{AD}$ patients incurred significantly elevated health care costs, more so in the untreated cohort, although not statistically significant (mean $\$ 7,306$ versus $\$ 6,308 ; p=0.1059$ ). Inpatient and post-acute SNF care were the main cost drivers of the Medicare expenditures incurred around the time of diagnosis. Inpatient spending increased significantly from the month leading up to diagnosis to the month of $\mathrm{AD}$ diagnosis (untreated: from $\$ 334$ to $\$ 3,039$; treated: from $\$ 86$ to $\$ 2,599$; both $p<0.0001)$; spending also increased for post-acute SNF expenditure (untreated: $\$ 197$ to $\$ 1,774$; treated: $\$ 93$ to $\$ 1,774$; all $p<0.0001$ ).

The average annual total Medicare expenditures (Fig. 3) were significantly higher among untreated patients $(\$ 30,110$ versus $\$ 25,828 ; p=0.0162)$, driven mostly by hospice care (cost difference $=\$ 2,658$; $p<0.0001)$. Inpatient and post-acute SNF costs were important cost drivers; however, the cost differences in inpatient ( $\$ 658 ; p=0.4252)$, and post-acute SNF $(\$ 1,124 ; p=0.161)$ were not statistically significant between untreated and treated patients. AD-related health care costs accounted for $43 \%$ (untreated) and $48 \%$ (treated) of the total Medicare expenditures. Treated patients compared to untreated patients had significantly higher AD-related office costs $(\$ 457$ versus $\$ 274 ; p<0.0001$ ). However, $\mathrm{AD}-$ related hospice care and associated costs $(\$ 2,452$ versus $\$ 322$; $p<0.0001)$ were significantly higher in the untreated cohort.

A similar trend was observed in terms of health care utilizations. Compared to treated patients, untreated patients had significantly more hospice visits $(0.76$ versus $0.17 ; p<0.0001)$. On the other hand, there were more outpatient office (14.88 versus $13.19 ; p=0.0148)$ and pharmacy (31.79 versus $29.44 ; p=0.0493)$ visits in treated patients compared to untreated patients (Supplementary Table 5). AD-related health care utilizations (Supplementary Table 5) were substantially higher in treated patients compared to untreated patients; except for hospice care visits $(0.57$ versus $0.10 ; p<0.0001)$ which were higher in the untreated cohort.

\section{DISCUSSION}

This retrospective analysis was conducted to evaluate overall survival, rate of institutionalization, and economic burden of treated versus untreated patients newly diagnosed with AD. After controlling for patient characteristics including comorbidities, we 


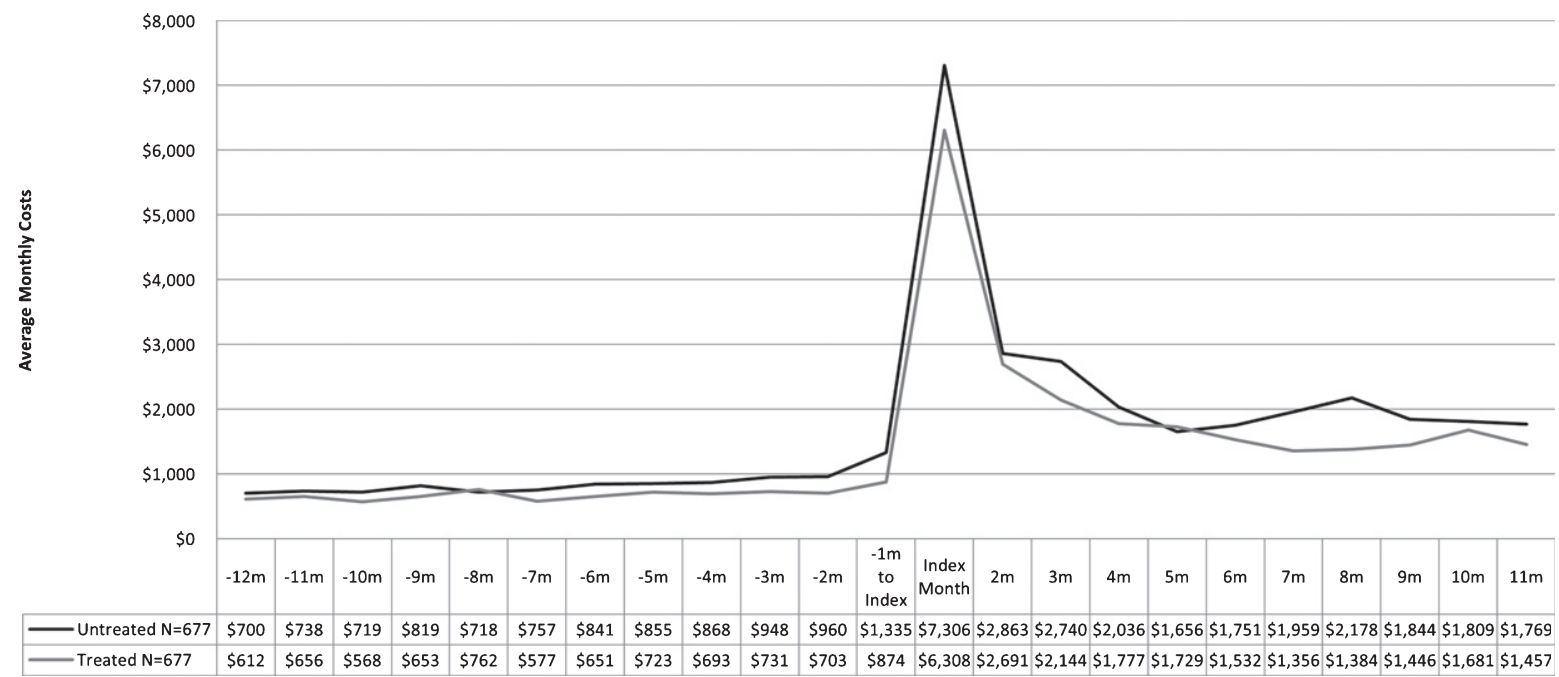

Fig. 2. PSM-adjusted all-cause average monthly cost among newly-diagnosed AD patients (economic analysis population). PSM, propensity score matching model adjusting for age, race, gender, geographic region, Charlson Comorbidity Index score, baseline all-cause health care utilization, baseline diagnostic tests (CT and MRI), and prevalence of individual comorbidities including Parkinson's disease, epilepsy, mood disorder, pneumonia, and mild cognitive impairment.

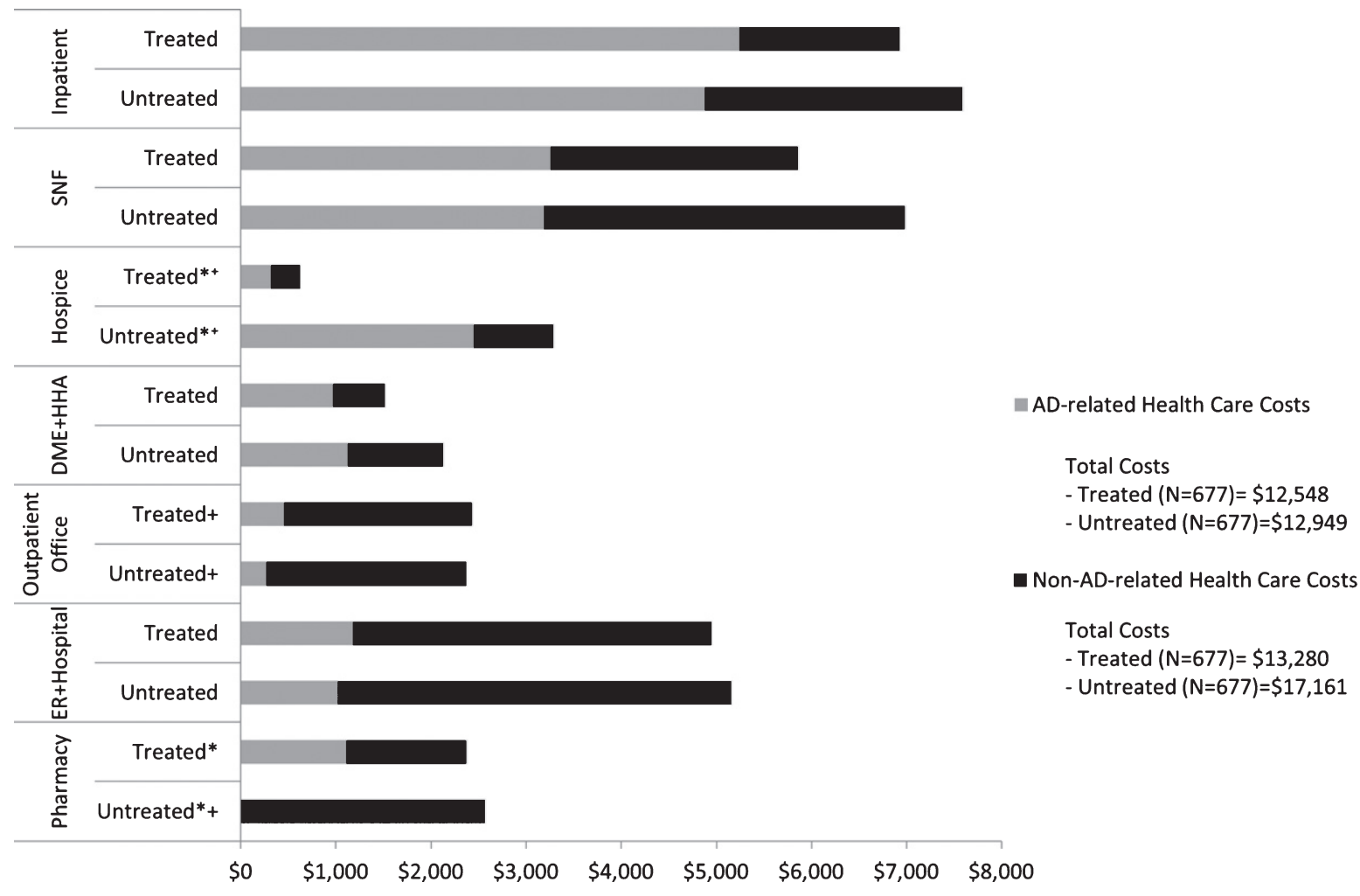

Fig. 3. Post-index PSM-adjusted annual all-cause and AD-related health care costs among newly-diagnosed AD patients (economic analysis population). AD, Alzheimer's disease; DME, durable medical equipment; ER, emergency room; HHA, home health agency; PSM, propensity score matching model adjusting for age, race, gender, geographic region, Charlson Comorbidity Index score, baseline all-cause health care utilization, baseline diagnostic tests (CT and MRI), and prevalence of individual comorbidities including Parkinson's disease, epilepsy, mood disorder, pneumonia, and mild cognitive impairment. ${ }^{*} p<0.05$ treated versus untreated for all-cause health care costs; ${ }^{+} p<0.05$ treated versus untreated for AD-related cause health care costs. 
observed that newly-diagnosed $\mathrm{AD}$ patients who received treatment had a lower risk of dying and being institutionalized compared to untreated counterparts. In addition, they incurred lower overall expenditures of approximately $\$ 4,282$ in total Medicare spending for the 12 months post-AD diagnosis compared to untreated patients. However, total AD-related costs were only slightly lower for the treated cohort, and the difference was not statistically significant. In addition, a large portion of the AD-related costs incurred by the untreated $\mathrm{AD}$ patients was due to their $\mathrm{AD}$ related hospice care. Previous studies showed that chronically ill patients with the ability and willingness to manage their health and care are more likely to adhere to or initiate treatment and, as a result, incur lower health care costs $[13,14]$. It is difficult to infer that the improved survival and lower institutionalization rates were solely due to AD treatment administration. However, the observed benefit from the study findings suggests that receiving $\mathrm{AD}$ treatment could be considered as a proxy for better quality of care, access to care, and improved health because patients who received AD therapy also most likely received consistent medical attention for other severe comorbidities. In general, AD patients get sicker faster due to their condition, and the cost of treating multiple complicated comorbidities results in overall higher spending $[15,16]$.

Early detection of dementia is beneficial to provide timely care and offer direct benefits to patients with dementia [17]. However, our study showed that close to $11 \%$ of untreated patients and approximately $4.3 \%$ of treated patients were institutionalized at time of the $\mathrm{AD}$ diagnosis. In addition, $67.8 \%$ of untreated $\mathrm{AD}$ patients were diagnosed in ambulatory settings compared to $81.2 \%$ of treated AD patients. This finding is consistent with previous reports [18], implying that many patients may receive their first AD diagnosis when they meet a health care professional as a result of another more serious health event. Furthermore, untreated patients had lower numbers of physician office and pharmacy visits, indicating a possible failure of ambulatory care management, which echoed prior study findings [19, 20].

The current study found that Medicare total costs in newly-diagnosed AD patients peaked significantly during the month of diagnosis, and AD-related costs accounted for $43-48 \%$ of the total Medicare expenditures. This finding is similar to those of other studies that examined the diagnostic pathways to AD10 and Medicare expenditures for patients with $\mathrm{AD}$ and AD-related dementia or MCI [21]. The major cost components of Medicare expenditures were found to be the use of inpatient and post-acute SNF services post-AD diagnosis in our analysis. Studies on hospital data have suggested that early and timely detection of $\mathrm{AD}$ can be cost-saving $[6,10,22]$.

There were several limitations in this study. By using retrospective claims data to identify newlydiagnosed $A D$ patients, these patients may represent individuals across the spectrum of $\mathrm{AD}$ severity and may not reflect results for patients with new onset of $\mathrm{AD}$. In addition, measures such as AD duration, disease severity and progression, cause of death, patient health behaviors, and caregiver information were not available in the Medicare data. Furthermore, non-pharmacological treatment information including computer cognitive training, diet, and regular moderate exercise were unavailable in the claims. Therefore, these estimates were not measured in our analysis. The lack of disease severity data can lead to potential underestimation of the severity of the disease of interest. However, we controlled for the differences between the treated and untreated cohorts using PSM, which created similar cohorts with well-balanced baseline demographic and clinical characteristics.

Services reimbursed by Medicaid, such as custodial care for patients who resided in a nursing facility for more than 100 days, are not reflected in this analysis. Moreover, this study included fee-for-service patients who had continuous enrollment in Medicare Parts A and B, which may limit the generalizability of the study results since patients enrolled in Medicare Part $\mathrm{C}$ were not included.

A strict and robust inclusion criterion was applied to capture newly-diagnosed AD patients by excluding patients who had an $\mathrm{AD}$ or dementia-related diagnosis as well as patients who were prescribed AD medications prior to the first index AD diagnosis. Although many patients receive AD treatment without a diagnosis, this analysis assessed newlydiagnosed $\mathrm{AD}$ patients. Prior $\mathrm{AD}$ or AD-related diagnosis was the cause for most patient exclusions $(80 \%)$. The exclusion criterion was applied in order to omit patients whose $\mathrm{AD}$ diagnosis may have manifested from other comorbid diagnoses.

Since Medicare is the primary payer for elderly patients in the United States, the results from our analysis are expected to reflect the health care resource utilization and cost patterns of the elderly patient population with newly-diagnosed AD. 


\section{Conclusion}

For patients who were newly diagnosed with AD, initiation of treatment as close to diagnosis as possible may translate to improved survival, lower institutionalization rates, fewer resource utilizations, and lower health care expenditures. Patients with newlydiagnosed $\mathrm{AD}$ experience elevated health care costs, mostly driven by hospital and post-acute skilled nursing home visits during the period following diagnosis. This analysis suggests that early diagnosis, better treatment and care management, especially in an ambulatory setting, could be beneficial for AD patients, caregivers, and the health care system from both economic and clinical perspectives.

\section{ACKNOWLEDGMENTS}

This study was funded by Merck \& Co., Inc. Christopher M. Black, Rezaul K. Khandker and Baishali M. Ambegaonkar are employees of Merck \& Co., Inc., and Xiaohan Hu was an employee of Merck \& Co., Inc. at the time of the analysis. Lin Xie, M. Furaha Kariburyo, and Onur Baser are employees of STATinMED Research, which is a paid consultant to Merck \& Co., Inc. Howard Fillit, was a consultant for Merck \& Co., Inc.

Authors' disclosures available online (http://j-alz. com/manuscript-disclosures/17-0518r1).

\section{SUPPLEMENTARY MATERIAL}

The supplementary material is available in the electronic version of this article: http://dx.doi.org/ 10.3233/JAD-170518.

\section{REFERENCES}

[1] Alzheimer's Association (2016) 2016 Alzheimer's disease facts and figures. Alzheimers Dement 12, 459-509.

[2] Oboudiyat C, Glazer H, Seifan A, Greer C, Isaacson RS (2013) Alzheimer's disease. Semin Neurol 33, 313-329.

[3] Plassman BL, Langa KM, Fisher GG, Heeringa SG, Weir DR, Ofstedal MB, Burke JR, Hurd MD, Potter GG, Rodgers WL, Steffens DC, Willis RJ, Wallace RB (2007) Prevalence of dementia in the United States: The aging, demographics, and memory study. Neuroepidemiology $\mathbf{2 9}$, 125-132.

[4] The National Institute on Aging. Alzheimer's Disease Fact Sheet. http://www.nia.nih.gov/alzheimers/publication/ alzheimers-disease-fact-sheet. Updated July 25, 2017. Accessed August 13, 2017.

[5] Alzheimer's Association. Changing the Trajectory of Alzheimer's Disease: How a Treatment by 2025 Saves Lives and Dollars. http://www.alz.org/documents _custom/trajectory.pdf. Updated 2015. Published 2015. Accessed 21 October 2016.

[6] Bianchetti A, Scuratti A, Zanetti O, Binetti G, Frisoni GB, Magni E, Trabucchi M (1995) Predictors of mortality and institutionalization in Alzheimer disease patients 1 year after discharge from an Alzheimer dementia unit. Dementia 6, 108-112.

[7] Suehs BT, Davis CD, Alvir J, van Amerongen D, Pharmd NC, Joshi AV, Faison WE, Shah SN (2013) The clinical and economic burden of newly diagnosed Alzheimer's disease in a Medicare advantage population. Am J Alzheimers Dis Other Demen 28, 384-392.

[8] Zhao Y, Kuo TC, Weir S, Kramer MS, Ash AS (2008) Healthcare costs and utilization for Medicare beneficiaries with Alzheimer's. BMC Health Serv Res 8, 108.

[9] Bentkover J, Cai S, Makineni R, Mucha L, Treglia M, Mor V (2012) Road to the nursing home: Costs and disease progression among Medicare beneficiaries with ADRD. Am J Alzheimers Dis Other Demen 27, 90-99.

[10] Charlson M, Charlson R, Peterson JC, Marinopoulos SS, Briggs WM, Hollenberg JP (2008) The Charlson comorbidity index is adapted to predict costs of chronic disease in primary care patients. J Clin Epidemiol 61, 1234-1240.

[11] Morris JN, Fries BE, Mehr DR, Hawes C, Phillips C, Mor V, Lipsitz LA (1994) MDS Cognitive Performance Scale. J Gerontol 49, M174-M182.

[12] Morris JN, Fries BE, Morris SA (1999) Scaling ADLs within the MDS. J Gerontol A Biol Sci Med Sci 54, M546M553.

[13] Greene J, Hibbard JH (2012) Why does patient activation matter? An examination of the relationships between patient activation and health-related outcomes. J Gen Intern Med 27, 520-526.

[14] Hibbard JH, Greene J (2013) What the evidence shows about patient activation: Better health outcomes and care experiences; Fewer Data on Costs. Health Affairs 32, 207-214.

[15] Morrison RS, Siu AL (2000) Survival in end-stage dementia following acute illness. JAMA 284, 47-52.

[16] Scrutton J, Brancati CU (2016) Dementia and comorbidities: Ensuring parity of care. http://www.ilcuk.org.uk/ index.php/publications/publication_details/dementia_and_ comorbidities_ensuring_parity_of_care1. Published April 2016. Accessed 1 December 2016.

[17] Bradford A, Kunik ME, Schulz P, Williams SP, Singh H (2009) Missed and delayed diagnosis of dementia in primary care: Prevalence and contributing factors. Alzheimer Dis Assoc Disord 23, 306.

[18] Gilden DM, Kubisiak JM, Sarsour K, Hunter CA (2015) Diagnostic pathways to Alzheimer disease. Alzheimers Dis Disord 9, 330-337.

[19] Fillit H, Hill JW, Futterman R (2002) Health care utilization and costs of Alzheimer's disease: The role of comorbid conditions, disease stage, and pharmacotherapy. Fam Med 34, 528-535.

[20] Hill JW, Futterman R, Duttagupta S, Mastey V, Lloyd JR, Fillit H (2002) Alzheimer's disease and related dementias increase costs of comorbidities in managed Medicare. $\mathrm{Neu}$ rology $\mathbf{5 8}, 62-70$.

[21] Lin PJ, Zhong Y, Fillit HM, Chen E, Neumann PJ (2016) Medicare expenditures of individuals with Alzheimer's disease and related dementias or mild cognitive impairment before and after diagnosis. J Am Geriatr Soc 64, 1549-1557.

[22] Weimer DL, Sager MA (2009) Early identification and treatment of Alzheimer's disease: Social and fiscal outcomes. Alzheimers Dement 5, 215-226. 